\title{
Fiber MOPA Based Tunable Source for Terahertz Spectroscopy
}

\author{
Andrew Malinowski, Dejiao Lin, Shaif-ul Alam, Zhaowei Zhang, Morten Ibsen and David Richardson \\ Optoelectronics Research Centre, University of Southampton, Southampton, SO17 1BJ, United Kingdom \\ anm@orc.soton.ac.uk \\ John Young, Paul Wright, Krikor Ozanyan \\ School of Electrical and Electronic Engineering, University of Manchester, Manchester M13 9PL, United Kingdom \\ Mark Stringer, Bob Miles \\ Institute for Microwaves and Photonics, University of Leeds, Leeds LS2 9JT , United Kingdom
}

\begin{abstract}
We have developed a terahertz spectrometer based on difference frequency generation of beams from a fiber MOPA. We present some demonstration data on water vapor absorption lines.

OCIS codes: (060.2320) Fiber Optics Amplifiers and Oscillators; (300.6495) Spectroscopy, terahertz
\end{abstract}

\section{Introduction}

Pulsed fiber MOPAs have received increasing attention over the past decade offering potential advantages in terms of efficiency, compactness, reliability, ease of thermal management and high average power levels relative to existing pulsed laser approaches [1]. Such sources are ideal for a diverse range of frequency conversion applications offering novel system opportunities spanning the soft $\mathrm{X}$-ray to $\mathrm{THz}$ frequency regimes.

Here we demonstrate a novel $\mathrm{THz}$ spectrometer based on difference frequency generation (DFG) from a dual wavelength tunable fiber MOPA system. The system was designed to provide GHz resolution and a broad tuning range - 0.7-2.4 THz in the current configuration. The source also provides the ability to switch rapidly between two frequencies to facilitate real time $\mathrm{THz}$ tomography applications. We demonstrate its performance by measuring the absorption features of water vapor in air. The ultimate aim of the system is to investigate combustion products and temperature distribution in flames in turbines.

\section{System setup and experimental results}

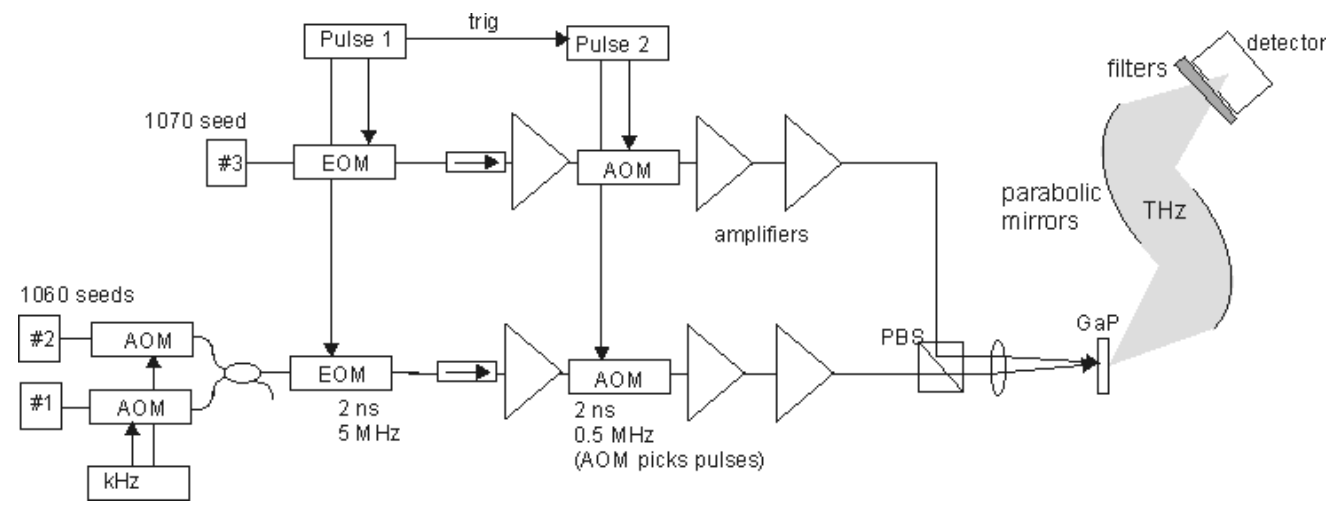

Fig. 1. The layout of the all-fiber Yb-doped MOPA system and THz generation and detection arrangement

Figure 1 shows the layout of our fiber MOPA system and the arrangement for generating and collecting $\mathrm{THz}$ radiation. Using two independent amplifier chains proves convenient for mixing the beams at a small angle (see below), as well as eliminating some deleterious nonlinear effects such as cross-phase modulation and four-wave mixing.

The seed lasers are strain tuned fiber DFB lasers with $\mathrm{kHz}$ linewidths. Rapid switching between seeds \#1, \#2 is possible using acousto-optic modulators as switches. Pulses of 2 ns duration are carved by electro-optic modulators. Using such short pulses eliminates stimulated Brilllouin scattering (SBS), a major power limitation in narrow linewidth fiber amplifier systems, which has a build-up time of a few nanoseconds. The synchronised pulses are carved with a $1 \%$ duty cycle, which is stepped down by pulse picking to $0.1 \%$ after the 1 st amplifier stage. 
The amplifier system uses PM fiber throughout. The final stages use commercial PM LMA fiber with a $25 \mu \mathrm{m}$ core diameter. The system is fully fiberised with the exception of the pump launch to the final stage, which is backward pumped via a free-space launch. The signal wavelengths are fed to the input of the final amplifier fibers by splicing directly to the tapered end of the LMA fiber. The system provides an average output power of up to $6 \mathrm{~W}$ per channel (pump limited) and a corresponding peak power of $6 \mathrm{~kW}$. The OSNR is $>25 \mathrm{~dB}$, the PER $>20 \mathrm{~dB}$ and the output is in a single transverse mode. Self-phase modulation in the amplifiers broadens the linewidths to $\sim 1 \mathrm{GHz}$.

The THz radiation is generated in a $2 \mathrm{~mm}$ thick GaP crystal. The crystal type and thickness were chosen to allow DFG over a broad bandwidth without realignment. GaP is isotropic and phase matching is achieved by non-collinear excitation [2]. The amplifier outputs are mixed with orthogonal polarisation using a PBS. There is a $\sim 0.3^{\circ}$ angle between the IR beams, resulting in a $\sim 40^{\circ}$ output angle between the IR and THz beams. The beams are focussed to a spot size of $\sim 300 \mu \mathrm{m}$ diameter, chosen to limit the divergence of the THz beam to the NA of the available optics. The generated THz is collected by means of gold parabolic mirrors and detected with a pyroelectric detector. A silicon filter prevents stray IR light reaching the detector. We obtained average THz powers of $\sim 1 \mu \mathrm{W}$ (peak power $1 \mathrm{~mW}$ ).
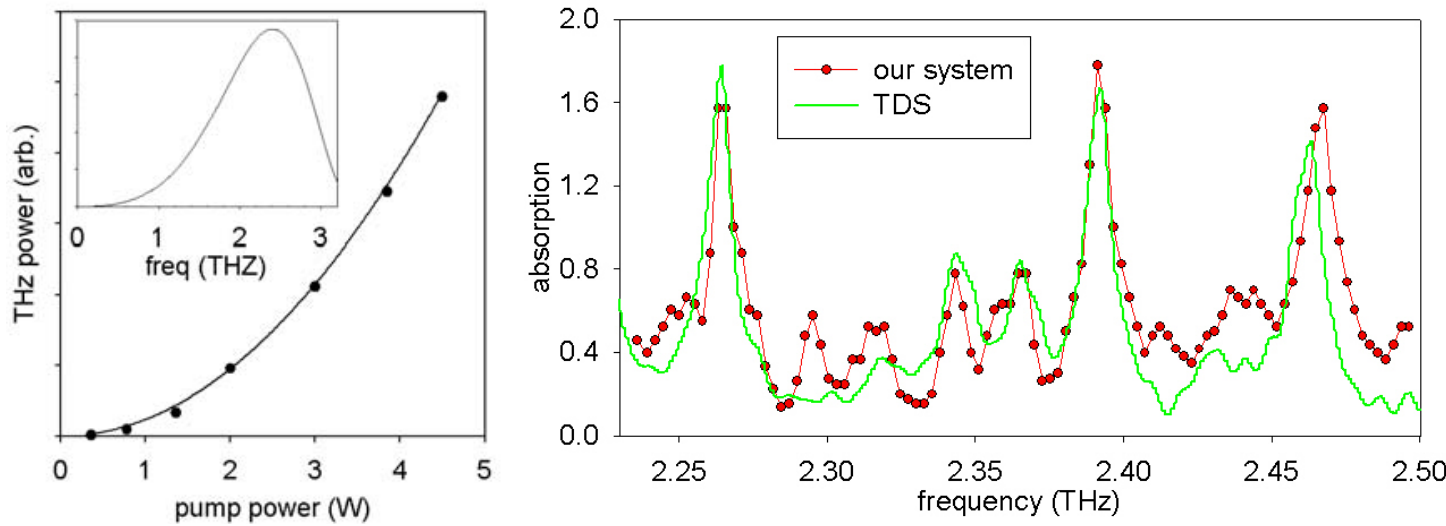

Fig. 2. a) THz emission as a function of laser power (fitted with a quadratic curve) with the inset showing the frequency dependence of the THz power calculated for our specific choice of non-collinear angle, b) Absorption spectrum in ambient air obtained by our system (line and circles) and with a TDS system (line) for comparison

The measured THz average power scales quadratically with pump power, as shown in Fig 2 a) and the inset shows that the THz generation efficiency peaks at $\sim 2.5 \mathrm{THz}$ in this arrangement, with a FWHM bandwidth of about $1 \mathrm{THz}$. As a demonstration of spectrometer performance we measured the water absorption lines in ambient air as shown in Fig. 2 b). The seed lasers used for this experiment had unstrained wavelengths $\lambda_{1}=\lambda_{2}=1060 \mathrm{~nm}, \lambda_{3}=1069$ $\mathrm{nm}$, yielding a difference frequency of $2.4 \mathrm{THz}$. Based on the bandwidths of the MOPAs, the spectral resolution should be less than $2 \mathrm{GHz}$. This is less than the width of the measured features $(\sim 10 \mathrm{GHz})$. We obtained an OSNR of $20 \mathrm{~dB}$ over a tuning range of $\sim 0.4 \mathrm{THz}$ (limited by the level of strain we were prepared to risk on the DFB fiber laser seeds). A trace obtained with a conventional TDS system is shown for comparison, showing good agreement between the two techniques. With other seed lasers and non-collinear angles we have demonstrated $\mathrm{THz}$ generation over the range 0.7-2.4 THz. We can switch rapidly between pairs of frequencies by differently straining DFBs \#1, \#2 and modulating their output with AOMs, opening a host of measurement opportunities.

\section{Conclusions}

We have successfully demonstrated a THz spectrometer based on DFG seeded by a fiber MOPA system. The fiber amplifiers have sufficient bandwidth to cover difference frequencies up to at least $5 \mathrm{THz}$. Rapid switching between two different $\mathrm{THz}$ frequencies for real-time tomography applications is possible simply by suitable gating of the three different seed diodes.

\section{References}

[1] D.J. Richardson et. al., "High power fiber lasers: current status and future perspectives,” JOSA_B 27, B63-B92 (2010).

[2] T. Tanabe, et. al., "Frequency-tunable terahertz wave generation via excitation of phonon-polaritons in GaP," Jnl. Of Physics D 36, 953-957 (2003). 